\title{
THE FAR-ULTRAVIOLET SPECTRUM OF $\gamma$ CASSIOPEIAE
}

\author{
DONALD C. MORTON, EDWARD B. JENKINS and RALPH C. BOHLIN \\ Princeton University Observatory, Princeton, N.J., U.S.A.
}

\begin{abstract}
The ultraviolet spectrum of $\gamma$ Cas from 1060 to $2120 \AA$ is described.
\end{abstract}
This is a brief report of the ultraviolet spectrum of $\gamma$ Cas obtained on November 15 , 1968 with the Princeton all-reflective rocket spectrograph. This star is famous for its sudden brightening from 2.2 to 1.6 magnitudes in 1936-37 and the accompanying appearance of pronounced shell lines. Since then the star has been relatively quiet until 1966, when Shelus (1967) observed that a veiling of the spectrum had occurred, filling in most of the visual absorption lines. $\mathrm{H} \alpha, \beta$, and $\gamma$ currently show emission profiles with double pzaks.

The rocket spectrum has about $1 \AA$ resolution from 1060 to $2120 \AA$. The principal absorption features are the interstellar Lyman $\alpha$ line and stellar lines of N II (1085 $\AA$ ),

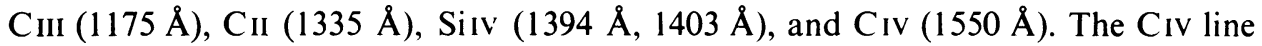
has an emission component on its long wavelength edge, and $C_{\text {II }}$ may have weak emission at both edges of the absorption feature.

The interstellar line is $8 \AA$ wide corresponding to $1.2 \times 10^{20}$ hydrogen atoms $\mathrm{cm}^{-2}$ in the line of sight for broadening by pure radiation damping. If we adopt a distance of $200 \mathrm{pc}$ to $\gamma$ Cas, the average volume density is only 0.2 atoms $\mathrm{cm}^{-3}$, similar to the low densities reported in the direction of Orion (Jenkins and Morton, 1967) and $\zeta$ Pup (Morton et al., 1969).

A preliminary analysis of the ultraviolet stellar spectrum has yielded two immediate results. First, there are no outstanding emission lines in this wavelength region. Even the $C_{I I}$ and $C$ IV lines are not as strong relative to the continuum as the $\mathrm{H} \beta$ emission. Secondly, there are no significant velocity shifts except for the CIV absorption line, which may be displaced about $400 \mathrm{~km} \mathrm{sec}^{-1}$ towards the observer. The spectrum is rather similar to the normal main-sequence stars $\delta$ Sco (B0V) and $\pi$ Sco (BIV) reported by Morton and Spitzer (1966), though some of the weaker lines found in the Scorpius stars seem to be absent in $\gamma$ Cas, probably as a result of the latter exposure having more background fog and poorer resolution.

\section{Acknowledgements}

This research was supported by contract NSr-31-001-901 with the United States National Aeronautics and Space Administration. A detailed report will be submitted to the Astrophysical Journal by R. Bohlin.

\section{References}

Jenkins, E. B. and Morton, D. C.: 1967, Nature 215, 1257.

Houziaux and Butler (eds.). Ultraviolet Stellar Spectra and Ground-Based Observations, 178-179. All Rights Reserved. Copyright (c) 1970 by the IAL'. 
Morton, D. C. and Spitzer, L.: 1966, Astrophys. J. 144, 1.

Morton, D. C., Jenkins, E. B., and Brooks, N. H.: 1969, Astrophys. J. 155, 875.

Shelus, P. J.: 1967, Sky and Telescope 33, 220.

\section{Discussion}

Houziaux: As additional information, I may say that we recently observed the spectrum of $\gamma$ Cas. at the Observatoire de Haute-Provence, at $20 \AA / \mathrm{mm}$. Double broad emissions of Paschen lines are seen as well as a strong emission line of Or at $8446 \AA$. 